CERN-EP/2000-154

December 13, 2000

\title{
Observation of weak neutral current neutrino production of $\mathrm{J} / \psi$
}

The CHORUS Collaboration

E. Eskut, A. Kayis-Topaksu, G. Onengüt

Çukurova University, Adana, Turkey

R. van Dantzig, M. de Jong, J. Konijn, O. Melzer, R.G.C. Oldeman, E. Pesen, C.A.F.J. van der Poel, J.W.E. Uiterwijk, J.L. Visschers

NIKHEF, Amsterdam, The Netherlands

M. Guler, M. Serin-Zeyrek, R. Sever, P. Tolun, M.T. Zeyrek

METU, Ankara, Turkey

N. Armenise, F. Cassol ${ }^{1}$, M.G. Catanesi, M. De Serio, M.T. Muciaccia, E. Radicioni, S. Simone, L. Vivolo

Università di Bari and INFN, Bari, Italy

\author{
A. Bülte, K. Winter \\ Humboldt Universität, Berlin, Germany ${ }^{2}$
}

P. Annis ${ }^{3}$, R. El-Aidi, M. Vander Donckt ${ }^{4}$, B. Van de Vyver ${ }^{5}$, P. Vilain ${ }^{6}$, G. Wilquet ${ }^{6}$

Inter-University Institute for High Energies (ULB-VUB) Brussels, Belgium

P. Righini ${ }^{7}$, B. Saitta

Università di Cagliari and INFN, Cagliari, Italy 


\section{E. Di Capua}

Università di Ferrara and INFN, Ferrara, Italy

Y. Ishii $^{8}$, T. Kawamura ${ }^{9}$, M. Kazuno, S. Ogawa, H. Shibuya

Toho University, Funabashi, Japan

J. Brunner ${ }^{1}$, M. Chizhov ${ }^{10}$, D. Cussans ${ }^{11}$, M. Doucet, J.P. Fabre, W. Flegel, M. Litmaath ${ }^{12}$, H. Meinhard, E. Niu ${ }^{13}$, H. Øverảs, J. Panman, I.M. Papadopoulos, S. Ricciardi ${ }^{14}$, A. Rozanov ${ }^{1}$, D. Saltzberg ${ }^{15}$, P. Strolin ${ }^{16}$, R. Tsenov ${ }^{10}$, C. Weinheimer ${ }^{17}$,

H. Wong ${ }^{18}$, P. Zucchelli ${ }^{19}$

CERN, Geneva, Switzerland

\section{J. Goldberg}

Technion, Haifa, Israel

M. Chikawa

Kinki University, Higashiosaka, Japan

E. Arik, A.A. Mailov

Bogazici University, Istanbul, Turkey

I.G. Park, J.S. Song, C.S. Yoon

Gyeongsang National University, Jinju, Korea

K. Kodama, N. Ushida

Aichi University of Education, Kariya, Japan

S. Aoki, T. Hara

Kobe University, Kobe, Japan

G. Brooijmans ${ }^{4,12}$, D. Favart, G. Grégoire, J. Hérin ${ }^{4}$

Université Catholique de Louvain, Louvain-la-Neuve, Belgium

A. Artamonov, P. Gorbunov, V. Khovansky, V. Shamanov, I. Tsukerman 
D. Bonekämper, N. D’Ambrosio, D. Frekers, D. Rondeshagen, T. Wolff ${ }^{20}$

Westfälische Wilhelms-Universität, Münster, Germany ${ }^{2}$

K. Hoshino, M. Komatsu, Y. Kotaka, T. Kozaki, M. Miyanishi, M. Nakamura, T. Nakano, K. Niu, K. Niwa, Y. Obayashi ${ }^{21}$, O. Sato, T. Toshito ${ }^{21}$

Nagoya University, Nagoya, Japan

N. Bruski, S. Buontempo, A.G. Cocco, G. De Lellis ${ }^{22}$, F. Di Capua, A. Ereditato, G. Fiorillo, R. Listone, M. Messina, P. Migliozzi, V. Palladino, V. Tioukov Università Federico II and INFN, Naples, Italy

K. Nakamura, T. Okusawa

Osaka City University, Osaka, Japan

A. Capone, D. De Pedis, S. Di Liberto, U. Dore, P.F. Loverre, L. Ludovici, A. Maslennikov ${ }^{23}$, G. Piredda, G. Rosa, R. Santacesaria, A. Satta, F.R. Spada

Università La Sapienza and INFN, Rome, Italy

E. Barbuto, C. Bozza, G. Grella, G. Romano, S. Sorrentino

Università di Salerno and INFN, Salerno, Italy

Y. Sato, I. Tezuka

Utsunomiya University, Utsunomiya, Japan

\begin{abstract}
Observation of $\mathrm{J} / \psi$ production by neutrinos in the calorimeter of the CHORUS detector exposed to the CERN SPS wide-band $\nu_{\mu}$ beam is reported. A spectrum-averaged crosssection $\sigma^{\mathrm{J} / \psi}=(6.3 \pm 3.0) \times 10^{-41} \mathrm{~cm}^{2}$ is obtained for $20 \mathrm{GeV} \leq E_{\nu} \leq 200 \mathrm{GeV}$. The data are compared with the theoretical model based on the QCD Z-gluon fusion mechanism.
\end{abstract}




\section{Introduction}

Open charm production in neutrino charged-current interactions has been studied in detail during the past three decades by several groups (see, for example, Ref. [1]). In particular, the CDHS [2], CHARM-II [3], CCFR [4], and NuTeV [5] experiments each have collected thousands of events initiated by this process, with a crosssection amounting to a few per cent of the total $\nu \mathrm{CC}$ cross-section. In neutralcurrent interactions, c-quarks appear only in pairs and, therefore, a much smaller production rate is expected. In these reactions the production of $\mathrm{J} / \psi$ decaying into a muon pair has the cleanest experimental signature. Evidence for this rare process was reported by CDHS about 20 years ago [6].

The $\mathrm{J} / \psi$ state can be produced either directly or via cascade decays of heavier charmonia: $\chi_{\mathrm{c} 1}, \chi_{\mathrm{c} 2} \rightarrow \gamma \mathrm{J} / \psi$ and $\psi^{\prime} \rightarrow \pi \pi \mathrm{J} / \psi$ (Fig. 1). Theoretical calculations of the cross-section of the direct $\mathrm{J} / \psi$ production by neutrinos $[7,8]$ were made in the framework of QCD-based Z-gluon fusion and vector dominance (VDM) models. In $\mathrm{VDM}$, only the vector coupling, $g_{\mathrm{V}}$, of the $\mathrm{Z}$ boson to the c-quark contributes, while in the Z-gluon fusion approach both the vector and the axial vector couplings, $g_{\mathrm{A}}$, are at work. One would expect the Z-gluon fusion mechanism to dominate because of the numerical smallness of $g_{\mathrm{V}}$ in the standard electroweak theory at $\sin ^{2} \theta_{\mathrm{W}}=$

1 Now at CPPM CNRS-IN2P3, Marseille, France.

2 Supported by the German Bundesministerium für Bildung und Forschung under contract numbers 05 6BU11P and 05 7MS12P.

3 Supported by Regione autonoma della Sardegna, Italy.

4 Institut Interuniversitaire des Sciences Nucléaires, Belgium.

5 Nationaal Fonds voor Wetenschappelijk Onderzoek, Belgium.

6 Fonds National de la Recherche Scientifique, Belgium.

7 Now at University of Bari, Bari, Italy.

8 Deceased.

9 Now at University of Naples "Federico II" and INFN, Naples, Italy.

${ }^{10}$ On leave of absence from St.Kliment Ohridski University of Sofia, Bulgaria.

${ }^{11}$ Now at University of Bristol, Bristol, UK.

12 Now at Fermi National Accelerator Laboratory, Batavia, IL, USA.

${ }^{13}$ Now at Center for Chronological Research, Nagoya University, Nagoya, Japan.

${ }^{14}$ Now at Royal Holloway College, University of London, Egham, UK.

${ }^{15}$ Now at U.C.L.A., Los Angeles, USA.

${ }^{16}$ On leave of absence from University of Naples "Federico II", Naples, Italy.

${ }^{17}$ Now at University of Mainz, Mainz, Germany.

${ }^{18}$ Now at Academia Sinica, Taipei, Taiwan.

${ }^{19}$ On leave of absence from INFN, Ferrara, Italy.

${ }^{20}$ Supported by a grant from Deutsche Forschungsemeinschaft.

${ }^{21}$ Now at Kamioka Observatory, Institute for Cosmic Ray Research, University of Tokyo, Gifu, Japan.

${ }^{22}$ Partially supported by the "Fondo Sociale Europeo".

${ }^{23}$ CASPUR, Rome, Italy. 

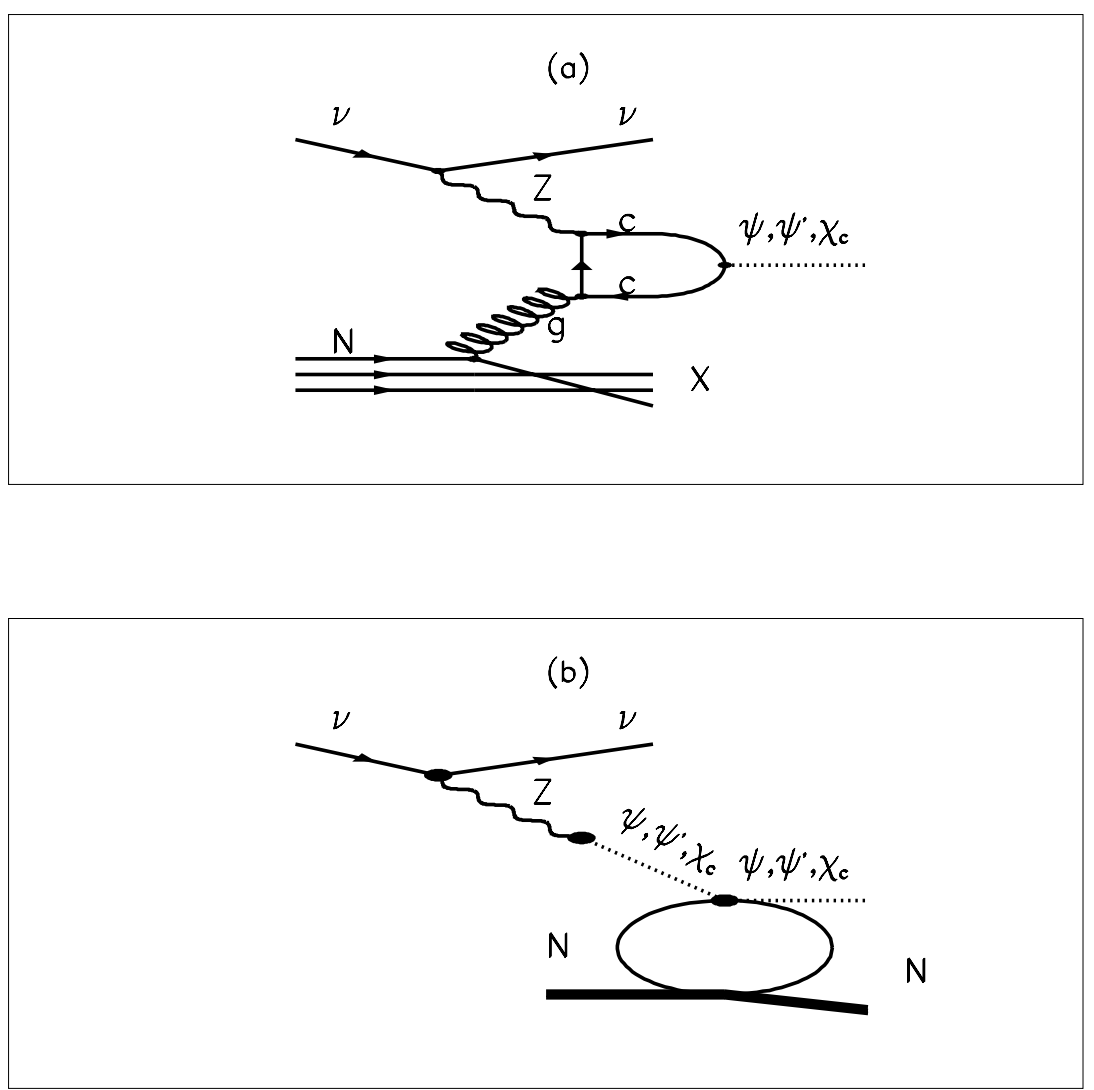

Fig. 1. Feynman diagrams for production of charmonium states in weak neutral-current interactions: (a) Z-gluon fusion model, (b) VDM model (diffraction).

0.23: $g_{\mathrm{V}}^{2} \approx 0.13\left(g_{\mathrm{V}}^{2}+g_{\mathrm{A}}^{2}\right)$. There are no predictions for the indirect $\mathrm{J} / \psi$ production rate in this model. Recent estimates based on the non-relativistic QCD approach [9] as well as generalized VDM calculations [8] show that the contributions of direct and indirect $\mathrm{J} / \psi$ production can be comparable. The overall $\mathrm{J} / \psi$ production rate is expected at the level of $3 \times 10^{-3}$ of the open charm production. 
The CHORUS experiment [10] searching for $\nu_{\mu} \rightarrow \nu_{\tau}$ oscillations in the CERN SPS wide-band $\nu_{\mu}$ beam recorded about five million dimuon triggers in its massive calorimeter. A muon spectrometer, located downstream from the calorimeter, is well suited to identify muons and to measure their momenta, thus allowing a search of the $\mathrm{J} / \psi \rightarrow \mu^{+} \mu^{-}$signal in these data to be undertaken. The experimental signature for $\mathrm{J} / \psi$ is an excess of events at the $\mathrm{J} / \psi$ mass in the invariant-mass distribution for the muon pairs.

The paper is organized as follows: in Section 2 the experimental set-up is described; Section 3 is devoted to the data analysis and $\mathrm{J} / \psi$ selection; and in Section 4 the $\mathrm{J} / \psi$ production cross-section is evaluated.

\section{The experimental set-up}

We have searched for $\mathrm{J} / \psi$ production in the CHORUS detector [10] exposed to the wide-band neutrino beam produced by $450 \mathrm{GeV}$ protons from the CERN SPS. The average $\nu_{\mu}$ energy is $27 \mathrm{GeV}$. The background of $\bar{\nu}_{\mu}$ is about $6 \%$ [10]. The 112-ton lead-scintillator calorimeter [11] was used as an active target for the $\mathrm{J} / \psi$ search instead of the 770-kg emulsion used as a target for neutrino oscillation searches. It provides the reconstruction of the event vertex, as well as the measurement of hadronic shower energy. The calorimeter consists of three sections with planes perpendicular to the beam direction and with decreasing granularity along the beam direction: EM (4 planes), HAD1 (5 planes) and HAD2 (5 planes). The depths of these sections are $16 \mathrm{~cm}, 40 \mathrm{~cm}$ and $50 \mathrm{~cm}$ respectively giving in total 5.2 interaction lengths. The tracking is provided by planes of limited streamer tubes between the calorimeter planes.

The rôle of the muon spectrometer $[10,12]$ located downstream from the calorimeter is to identify muons and to determine their trajectory, momentum and charge. It consists of six magnetized iron toroids, instrumented with scintillators, and tracking detectors composed of drift chambers and limited streamer tubes. Muon momenta are determined from their curvature in the toroidal magnetic field. The momentum resolution is limited mainly by multiple Coulomb scattering in the iron. It is about $15 \%$ [12] in the region of $12 \mathrm{GeV}$ to $28 \mathrm{GeV}$ and $\approx 19 \%$ at $71 \mathrm{GeV}$ [10] as measured with test-beam muons. For tracks with $p_{\mu} \leq 4.5 \mathrm{GeV}$, stopping inside the spectrometer, the momentum can be reconstructed also by range with $\approx 6 \%$ precision [10]. The muon spectrometer also provides rough measurement of the shower energy leakage from the calorimeter.

The trigger system [13] of the CHORUS experiment has different types of trigger for events originating from the emulsion, the calorimeter and the muon spectrometer. For our purpose we use the dimuon trigger, which requires a double-hit pattern in the calorimeter or in the muon spectrometer and an activity in the first two mag- 
nets of the muon spectrometer.

\section{The analysis}

For the analysis we used the standard CHORUS reconstruction program CHORAL [14]. In total, 4.7 million events recorded with the dimuon trigger were processed. This sample corresponds to $\approx 4.2 \times 10^{19}$ protons on the neutrino target. Most of these events contain one track and an energetic hadronic shower. Only $2.3 \times 10^{5}$ events have two reconstructed tracks with at least $2 \mathrm{GeV}$ momenta at the spectrometer entry and crossing at least two spectrometer magnets ( $\geq 1 \mathrm{~m}$ of iron) [15].

At the next step dimuons of opposite charge were selected. Muons were required to traverse at least four spectrometer magnets and be successfully reconstructed. The transverse distance between the tracks of $\mu^{+}$and $\mu^{-}$at the vertex plane was limited to $<15 \mathrm{~cm}$. The fiducial volume was chosen with a lateral size of $240 \times 240 \mathrm{~cm}^{2}$ and length of about $130 \mathrm{~cm}$ (the vertex should lie within the calorimeter planes from 3 to 11) to fulfil the requirement of the dimuon trigger. The fiducial target mass is 38 tons. To suppress the background from $\nu \mathrm{CC}$ events with $\pi^{+}\left(\mathrm{K}^{+}\right) \rightarrow \mu^{+} \mathrm{X}$ decays, both muons were required to have momenta above $5 \mathrm{GeV}$ at the vertex. They are calculated by adding the energy-dependent average muon energy losses in the calorimeter to the momenta reconstructed in the spectrometer [16]. The visible energy for the event, $E_{\nu}^{\mathrm{vis}}$, was required to be larger than $20 \mathrm{GeV}$. It is defined as the sum of muon momenta and shower energy deposited in the calorimeter and in the spectrometer: $E_{\mathrm{sh}}^{\mathrm{vis}}=E_{\mathrm{sh}}^{\mathrm{calo}}+E_{\mathrm{sh}}^{\mathrm{spec}}$. The muons were subtracted from the shower. In addition, we required $E_{\mathrm{sh}}^{\mathrm{spec}} / E_{\mathrm{sh}}^{\mathrm{vis}} \leq 0.3$ to reduce the effects due to the poor spectrometer energy resolution. $14995 \mu^{+} \mu^{-}$events survived all these cuts.

The distributions of the muon momenta and of the angle between muons in this sample are shown in Fig. 2. All these spectra are well described by GEANT 3.21 based Monte Carlo (MC) simulations ${ }^{24}$ of single charm production in the complete set-up, including modelling of the neutrino beam and the standard event reconstruction [19]. It should be noted that the MC does not include the background from $\nu \mathrm{CC}$ events with subsequent muonic decays of pions and kaons. Its contribution $(\approx 15 \%)$ was estimated from a $\mu^{-} \mu^{-}$subsample. The observed invariantmass distribution of $\mu^{+} \mu^{-}$pairs is presented in Fig. 3a. An approximation of the shape consists of $85 \%$ of the $\mu^{+} \mu^{-}$MC and of $15 \%$ of the $\mu^{-} \mu^{-}$data. The fit gives $\chi^{2} / N D F=1.31$. Taking into account that some instrumental effects were not included in the MC such an agreement is reasonable. ${ }^{25}$ Unfortunately, the MC

${ }^{24}$ For the simulation we used EHLQ structure functions [17] and Peterson longitudinal fragmentation function [18] for charmed quark $D(z) \propto z^{-1}\left(1-\frac{1}{z}-\frac{\epsilon \mathrm{P}}{1-z}\right)^{-2}$. The value of the parameter $\epsilon_{\mathrm{P}}$ was chosen to be 0.072 as obtained from the CHARM-II data [3].

${ }^{25}$ The fit quality is slightly better $\left(\chi^{2} / N D F=1.24\right)$ if we exclude the $\mu^{-} \mu^{-}$data. 

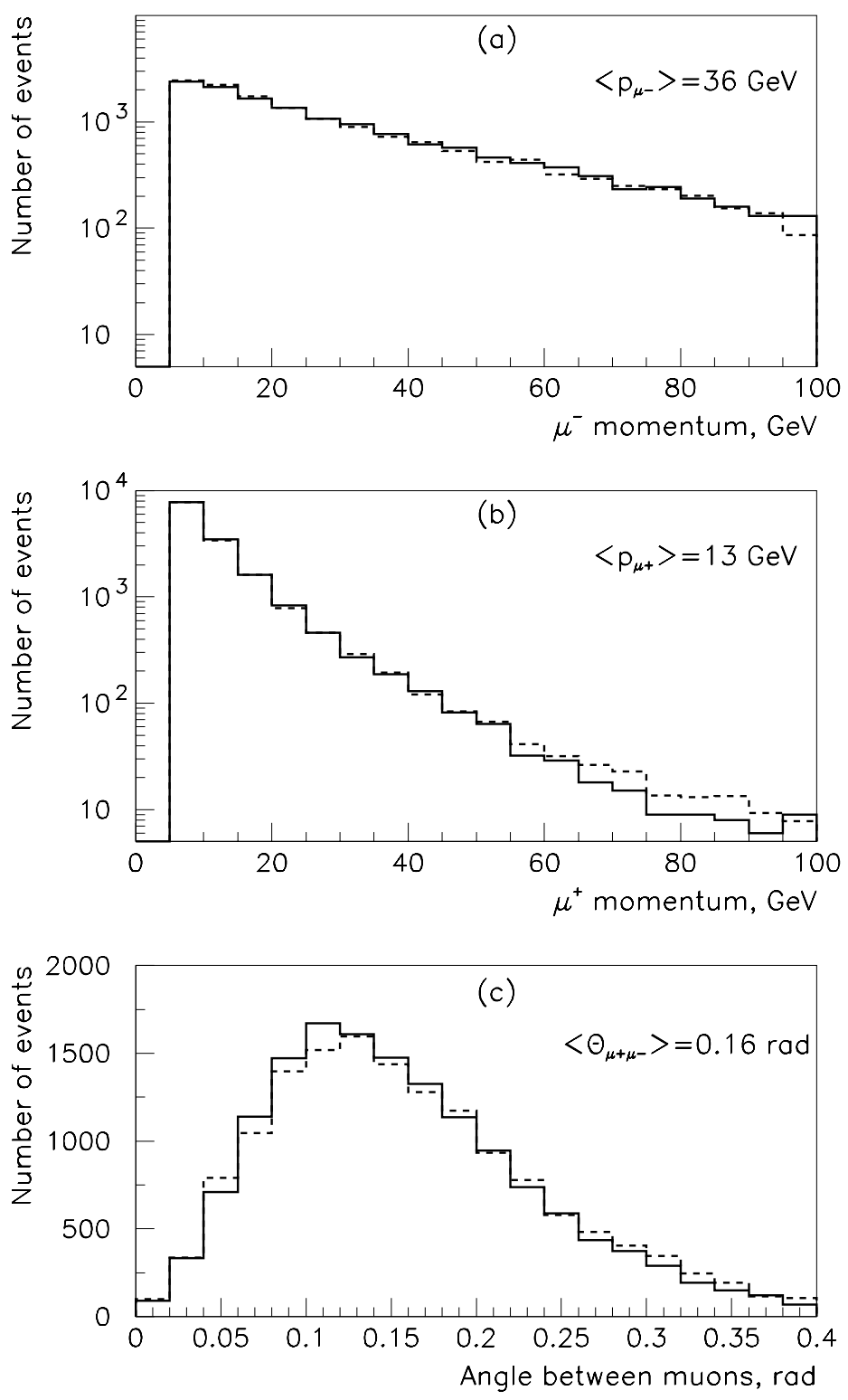

Fig. 2. Kinematical distributions of $\mu^{+} \mu^{-}$events (solid histograms are the data and dashed histograms are the Monte Carlo predictions): (a) $\mu^{-}$momentum; (b) $\mu^{+}$momentum; (c) the angle between $\mu^{-}$and $\mu^{+}$.

sample $(\approx 10000$ events $)$ is comparable to the data sample and not larger as the simulation procedure is very CPU time consuming. However, it is suitable for our goals which do not include a detailed study of single charm production. The observed average visible energy in the dimuon events, $\left\langle E_{\nu}^{\mathrm{vis}}\right\rangle \approx 85 \mathrm{GeV}$, corresponds to a total energy of about $100 \mathrm{GeV}$, i.e. twice the average energy of $\mathrm{CC}$ events. 
To reduce the background an upper cut on the shower energy was applied. $\mu^{+} \mu^{-}$ invariant-mass distributions were analysed at different limits on $E_{\mathrm{sh}}^{\mathrm{vis}}$. A structure appears in the $\mathrm{J} / \psi$ mass region at $E_{\mathrm{sh}}^{\mathrm{vis}} \leq 15 \mathrm{GeV}$. The excess of events in this region is clearly seen with the cut on the shower energy varying between 14 and $10 \mathrm{GeV}$. It is gradually reduced by further tightening the cut and vanishes at $E_{\mathrm{sh}}^{\mathrm{vis}} \leq$ $5 \mathrm{GeV}$. This is qualitatively in accordance with theoretical expectations [8] of a small diffractive ${ }^{26}$ part of the direct $\mathrm{J} / \psi$ production cross-section [8]. It should be noted that the cut $E_{\mathrm{sh}}^{\mathrm{vis}} \leq 5 \mathrm{GeV}$ could reject a significant fraction of events with $\mathrm{J} / \psi$ produced via cascade decays of excited charmonium states, $\chi_{\mathrm{c} 1}, \chi_{\mathrm{c} 2} \rightarrow \gamma \mathrm{J} / \psi$ and $\psi^{\prime} \rightarrow \pi^{+} \pi^{-} \mathrm{J} / \psi$, as photons and pions are expected to be in the energy range of several $\mathrm{GeV}$.

Figure $3 \mathrm{~b}$ shows the excess observed for $E_{\mathrm{sh}}^{\mathrm{vis}} \leq 10 \mathrm{GeV} .{ }^{27}$ The invariant-mass distribution of muon pairs in all $\mu^{+} \mu^{-}$events (Fig. 3a, solid histogram) was used as a background shape to the spectrum. In total, 1265 events survived the cut. To improve the signal-to-background ratio we applied the selection $p_{\mu^{+}} \geq p_{\mu^{-}}$. This muon momentum 'asymmetry' cut suppresses the background by a factor of about five as, on average, $\mu^{+}$is much softer than $\mu^{-}$(Fig. 2), whereas in $\mathrm{J} / \psi$ decays $p_{\mu^{+}} \approx p_{\mu^{-}}$. The resulting invariant-mass distribution is shown in Fig. 3c. In total, 226 events survived the cut. In the signal region between 2.75 and $3.75 \mathrm{GeV}$ there are 62 events. Again, the spectrum of all $\mu^{+} \mu^{-}$events was used as a background shape, with a small correction to account for the observed excess in the $\mathrm{J} / \psi$ region. It was normalized in the interval between 0 and $6 \mathrm{GeV}$, excluding the signal region. The fit gives $\chi^{2} / N D F=19.6 / 19$. The background $B$ is $42.5 \pm 7.3$ events. The error $\Delta B_{\text {stat }}$ includes both statistical fluctuation $(\sqrt{B})$ and normalization uncertainty (8\%) added in quadrature. The excess $S$ above the background is $19.5 \pm 8.6$ (stat) events. To evaluate the systematical error due to the background shape variation caused by $E_{\mathrm{sh}}^{\mathrm{vis}} \leq 10 \mathrm{GeV}$ and $p_{\mu^{+}} \geq p_{\mu^{-}}$cuts, we performed a particle-level MC study using about 500000 simulated dimuon events and a simplified description of the muon spectrometer. Our kinematical cuts and the procedure of background normalization and subtraction were applied to these simulated events. The average systematical uncertainty of the number of background events in the signal region was found to be $4.5 \%$. This corresponds to 1.9 events for our data sample.

Table 1 shows how the excess $S$, the background $B$ and its statistical error $\Delta B_{\text {stat }}$ depend on the upper cut on the shower energy $E_{\mathrm{sh}}^{\mathrm{vis}}$. It was checked with the binned likelihood method [21] that the normalization procedure produced unbiased estimates of $S$ and $B$ with all upper cuts on $E_{\mathrm{sh}}^{\mathrm{vis}}$ listed in Table 1.

\footnotetext{
${ }^{26}$ We follow the conventional terminology, applying the term 'diffractive' only to the VDM process (Fig. 1b). In our case this corresponds to a few $\mathrm{GeV}$ of $E_{\mathrm{sh}}^{\mathrm{vis}}\left(z \approx E^{\mathrm{J} / \psi} /\left(E^{\mathrm{J} / \psi}+\right.\right.$ $\left.E_{\mathrm{sh}}^{\mathrm{vis}}\right) \geq 0.9$ ), while in Ref. [6] the same term is applied for events surviving the cut $E_{\mathrm{sh}}^{\mathrm{vis}} \leq$ $10 \mathrm{GeV}$.

${ }^{27} \mathrm{CDHS}$ reported evidence for $\mathrm{J} / \psi$ production by neutrinos via neutral currents at the same upper cut on the shower energy [6].
} 

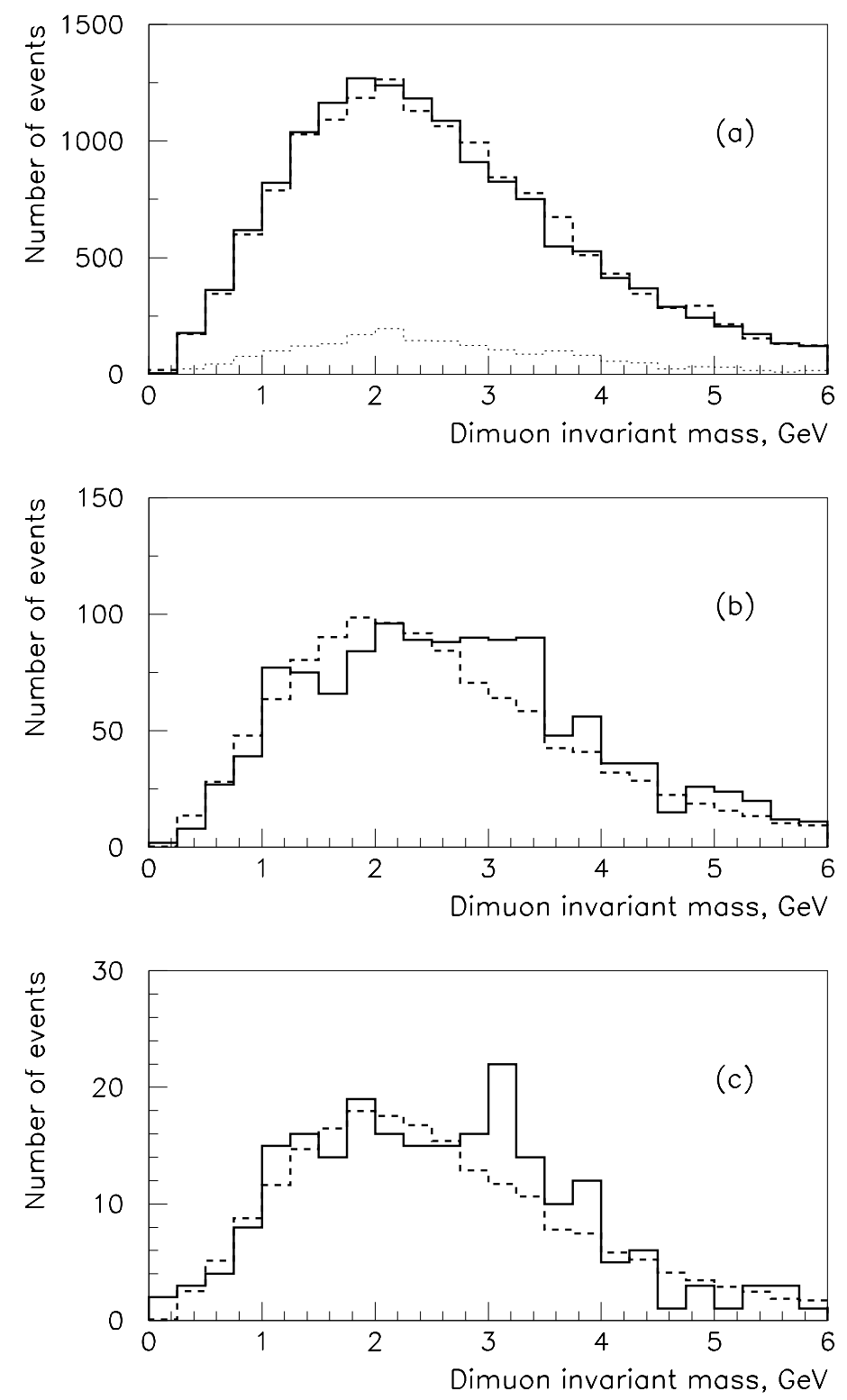

Fig. 3. The invariant-mass distributions for $\mu^{+} \mu^{-}$events: (a) all selected events (solid histogram is the data and dashed histogram is the sum of the Monte Carlo prediction and the $\mu^{-} \mu^{-}$data). The $\mu^{-} \mu^{-}$data alone is also shown (dotted histogram); (b) events with cut $E_{\mathrm{sh}}^{\mathrm{vis}} \leq 10 \mathrm{GeV}$ (solid histogram is the data and dashed histogram is the prediction for a background shape based on overall dimuon events); (c) events with cuts $E_{\mathrm{sh}}^{\mathrm{vis}} \leq 10 \mathrm{GeV}$ and $p_{\mu^{+}} \geq p_{\mu^{-}}$(solid histogram is the data and dashed histogram is the prediction for a background shape based on overall dimuon events). 
Table 1

Excess and background in the signal region at different upper cuts on $E_{\mathrm{sh}}^{\mathrm{vis}}(\mathrm{GeV})$.

\begin{tabular}{|c|c|c|c|c|c|c|c|c|c|c|c|}
\hline$E_{\mathrm{sh}}^{\mathrm{vis}} \leq$ & 5 & 6 & 7 & 8 & 9 & 10 & 11 & 12 & 13 & 14 & 15 \\
\hline$S$ & 1.0 & 8.6 & 7.2 & 8.7 & 9.7 & 19.5 & 24.8 & 24.0 & 21.8 & 23.6 & 23.4 \\
\hline$B$ & 8.0 & 12.4 & 18.8 & 25.3 & 34.3 & 42.5 & 52.2 & 63.0 & 77.2 & 88.4 & 99.6 \\
\hline$\Delta B_{\text {stat }}$ & 3.2 & 4.0 & 4.9 & 5.7 & 6.6 & 7.3 & 8.1 & 8.9 & 9.9 & 10.6 & 11.2 \\
\hline
\end{tabular}

The observed excess has to be corrected for finite invariant-mass resolution. The MC predicts the resolution of $0.41 \pm 0.02 \mathrm{GeV}$ at $\mathrm{J} / \psi$ mass. Our choice of the signal region was based on the MC optimization of the signal-to-background ratio for the given background shape. The correction factor of 1.44 has been applied to account for the signal tails outside of the signal region. The resulting total signal, thus, amounts to $N_{\mathrm{obs}}^{\mathrm{J} / \psi}=28.1 \pm 12.3$ (stat) \pm 2.7 (syst) events, with the cuts $E_{\mathrm{sh}}^{\mathrm{vis}} \leq$ $10 \mathrm{GeV}$ and $p_{\mu^{+}} \geq p_{\mu^{-}}$.

\section{$4 \mathrm{~J} / \psi$ production cross-section}

About one million $\mathrm{CC}$ events recorded in the calorimeter were used to normalize the neutrino flux by counting the number of events in bins of different energy and correcting for experimental effects. The total flux corresponds to more than 15 million physical CC events with $E_{\nu} \geq 20 \mathrm{GeV}$ in the fiducial volume.

To calculate the cross-section of $\mathrm{J} / \psi$ production in weak NC interactions, we use the following expression for the number of observed events:

$$
\begin{aligned}
N_{\mathrm{obs}}^{\mathrm{J} / \psi} & =\int N_{f . v .} B \sigma^{\mathrm{J} / \psi}\left(E_{\nu}\right) \epsilon\left(E_{\nu}\right) \phi_{\nu}\left(E_{\nu}\right) \mathrm{d} E_{\nu} \\
& =\frac{B}{\sigma_{0}^{\mathrm{CC}}}\left\langle\sigma^{\mathrm{J} / \psi}\right\rangle \int \epsilon\left(E_{\nu}\right) \frac{N^{\mathrm{CC}}\left(E_{\nu}\right)}{E_{\nu}} \mathrm{d} E_{\nu},
\end{aligned}
$$

where $N_{f . v}$. is the total number of nucleons in the fiducial volume, $B=\operatorname{Br}(\mathrm{J} / \psi \rightarrow$ $\left.\mu^{+} \mu^{-}\right)=0.06$ [20], $\quad \sigma_{0}^{\mathrm{CC}}=0.677 \times 10^{-38} \mathrm{~cm}^{2} \mathrm{GeV}^{-1}$ [1] is defined from the world-average total $\nu \mathrm{CC}$ cross-section on an isoscalar target $\left(\sigma_{\text {total }}^{\mathrm{CC}}=\sigma_{0}^{\mathrm{CC}} E_{\nu}\right)$, $\left\langle\sigma^{\mathrm{J} / \psi}\right\rangle$ is the $\mathrm{J} / \psi$ cross-section per nucleon averaged over the effective beam spectrum in the range of $20 \leq E_{\nu} \leq 200 \mathrm{GeV}, \epsilon\left(E_{\nu}\right)$ is the global $\mathrm{J} / \psi$ detection efficiency, $\phi_{\nu}\left(E_{\nu}\right)$ is the time-integrated total neutrino flux, and $N^{\mathrm{CC}}\left(E_{\nu}\right)$ is the time-integrated spectral density of physical CC events on an average nucleon [22].

The global efficiency consists of four factors: $\epsilon=\epsilon_{\text {det }} \epsilon_{\text {rec }} \epsilon_{\text {trig }} \epsilon_{\text {cut }}$. The detector efficiency, $\epsilon_{\text {det }}$, is defined as $\epsilon_{\text {det }}=\epsilon_{C H O R U S}\left(1-\epsilon_{\text {dead }}\right) \approx 0.87$, where $\epsilon_{C H O R U S} \approx$ 0.92 is the average CHORUS data collection efficiency [13], and $\epsilon_{\text {dead }} \approx 0.054$ is the average dead time during data-taking [23]. The reconstruction efficiency for 
$\mu^{+} \mu^{-}$events in the spectrometer, $\epsilon_{\mathrm{rec}} \approx 0.95 \pm 0.03$ [24], was evaluated by means of scanning by eye the subsample of the $\mu^{+} \mu^{-}$MC events [19]. The efficiency of the dimuon trigger, $\epsilon_{\text {trig }} \approx 0.80 \pm 0.11$ [24], was estimated with the MC. The energy dependence of the efficiency of the kinematical cuts, $\epsilon_{\text {cut }}\left(E_{\nu}\right)$, was calculated using the kinematics of direct $\mathrm{J} / \psi$ production and decay in the framework of the Zgluon fusion model. The differential cross-section for the process, $\mathrm{d}^{2} \sigma^{\mathrm{J} / \psi} / \mathrm{d} Q^{2} d \nu$, was taken from [8a]. ${ }^{28}$ The parametrization of the spectrometer muon momentum resolution from Ref. [10] was used. The smearing of the angle between muons due to multiple scattering in the calorimeter was also taken into account. The calculated $\epsilon_{\text {cut }}\left(E_{\nu}\right)$ is shown in Fig. 4a. The global efficiency was further folded with the neutrino beam spectrum (Fig. 4b). The average value of $\epsilon$ is about 0.18 .

From formula (1) the theoretical expectation [8] of direct $\mathrm{J} / \psi$ production in the framework of the Z-gluon fusion model is $8.0 \pm 1.5$ events. The main uncertainty comes from the efficiency calculation $(\sim 15 \%)$. Other sources give $\sim 10 \%$ contribution. The theoretical uncertainties are very difficult to calculate reliably and are not taken into account. A higher experimental rate $(28.1 \pm 12.3 \pm 2.7$ events $)$ suggests a sizeable contribution of excited charmonium states. We recall that it may be about as large as the direct $\mathrm{J} / \psi$ production cross-section (see Refs. [8,9]).

The experimental spectrum-averaged cross-section calculated with formula (1) is $\left\langle\sigma^{\mathrm{J} / \psi}\right\rangle=(6.3 \pm 3.0) \times 10^{-41} \mathrm{~cm}^{2} /$ nucleon. The error includes both statistical and systematical uncertainties added in quadrature. The CDHS result [6] is (5.4 \pm 1.9$)$ $\times 10^{-41} \mathrm{~cm}^{2} /$ nucleon ${ }^{29}$. It should be mentioned that CHORUS and CDHS were using different wide-band beams, with slightly different energy spectra and compositions. Although we do not expect large nuclear effects, we note that the CDHS experiment used an iron target, while CHORUS used a lead target.

In conclusion, the rare process of $\mathrm{J} / \psi$ production via neutrino neutral-current interaction has been observed. The measured cross-section is in agreement with the previous CDHS result [6]. The diffraction mechanism is unlikely to play an important rôle in the direct $\mathrm{J} / \psi$ production process. A contribution of excited charmonium states decaying to $\mathrm{J} / \psi$ could be as large as the direct $\mathrm{J} / \psi$ production process, in qualitative agreement with theoretical expectations. Therefore neutral-current coupling to charmed quarks cannot be extracted with sufficient precision.

\footnotetext{
${ }^{28}$ The main term in this expression is the virtual photoproduction cross-section which was chosen in the form $\sigma\left(\nu, Q^{2}\right)=\left(1+Q^{2} / M_{\mathrm{J} / \psi}^{2}\right)^{-2} A \exp (-B /(\nu-C))$ [25], with parameters $A=20 \mathrm{nb}, B=45 \mathrm{GeV}$ and $C=6 \mathrm{GeV}$ [26] tuned to $\mathrm{J} / \psi$ muoproduction data.

${ }^{29} \mathrm{CDHS}$ cross-section was rescaled from the published value of $(4.2 \pm 1.5) \times$ $10^{-41} \mathrm{~cm}^{2} /$ nucleon as the measured $\mathrm{J} / \psi$ branching ratio to muon pairs has changed from 0.07 to 0.06 and $\sigma_{0}^{\mathrm{CC}}$ has increased from 0.62 [27] to $0.677 \times 10^{-38} \mathrm{~cm}^{2} \mathrm{GeV}^{-1}$ [1] since 1982.
} 

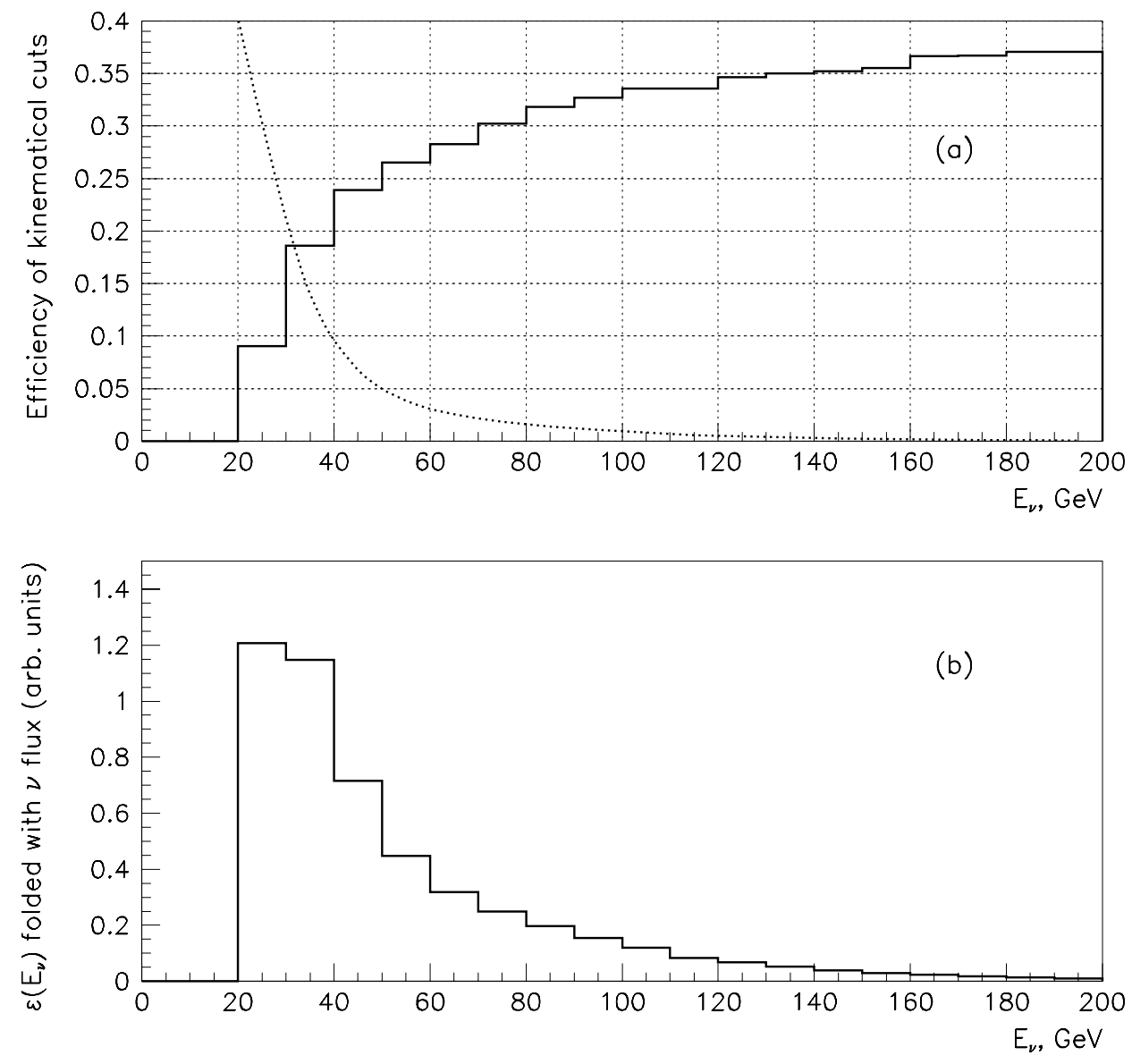

Fig. 4. (a) The efficiency of the kinematical cuts for direct $\mathrm{J} / \psi$ production as a function of $E_{\nu}$ (histogram). The neutrino spectrum is also shown (dotted curve). (b) The efficiency-weighted ('effective') neutrino spectrum.

\section{Acknowledgement}

We would like to thank A. Kaidalov, S. Kruchinin and V. Lemaitre for fruitful discussions. We gratefully acknowledge the help and support of our numerous tech- 
nical collaborators who contributed to the detector construction and operation. We thank the neutrino beam staff for their competent assistance, ensuring the excellent performance of the facility. The accumulation of a large data sample in this experiment was also made possible thanks to the efforts of the crew operating the CERN PS and SPS. The general technical support from EP, ECP and IT Divisions is gratefully acknowledged. We thank S. Anthony for help in preparation of the text.

The experiment has been made possible by grants from our funding agencies: the Institut Interuniversitaire des Sciences Nucléaires and the Interuniversitair Instituut voor Kernwetenschappen (Belgium); the Israel Science Foundation (Grant 328/94) and the Technion Vice President Fund for the Promotion of Research (Israel); CERN (Geneva, Switzerland); the German Bundesministerium für Bildung und Forschung (Grant 057MS12P(0)) (Germany); the Institute of Theoretical and Experimental Physics (Moscow, Russia); the Istituto Nazionale di Fisica Nucleare (Italy); the Promotion and Mutual Aid Corporation for Private Schools of Japan and Japan Society for the Promotion of Science (Japan); the Korea Research Foundation (Grant KRF-99-005-D00004) (Republic of Korea); the Foundation for Fundamental Research on Matter (FOM) and the National Scientific Research Organization (NWO) (The Netherlands); and the Scientific and Technical Research Council of Turkey (Turkey).

\section{References}

[1] J.M. Conrad, M.H. Shaevitz, and T. Bolton, Rev. Mod. Phys. 70 (1998) 1341.

[2] H. Abramowicz et al., CDHS Collaboration, Z. Phys. C15 (1982) 19.

[3] P. Vilain et al., CHARM II Collaboration, Eur. Phys. J. C11 (1999) 19.

[4] S. Rabinowitz et al., CCFR Collaboration, Phys. Rev. Lett. 70 (1993) 134;

A. Bazarko et al., CCFR Collaboration, Z. Phys. C65 (1995) 189.

[5] T. Adams et al., NuTeV Collaboration, Phys. Rev. D61 (2000) 092001.

[6] H. Abramowicz et al., CDHS Collaboration, Phys. Lett. B109 (1982) 115.

[7] V. Barger, W.Y. Keung, and R.J.N. Phillips, Phys. Lett. B92 (1980) 179.

[8] (a) J. Kühn and R. Rückl, Phys. Lett. B95 (1980) 431;

(b) R. Rückl, Preprint MPI-PAE/Pth 25/80, July 1980.

[9] A.A. Petrov, and T. Torma, Phys. Rev. D60 (1999) 093009.

[10] E. Eskut et al., CHORUS Collaboration, Nucl. Instrum. Methods A401 (1997) 7. 
[11] S. Buontempo et al., Nucl. Instrum. Methods A349 (1994) 70;

E.Di Capua et al., Nucl. Instrum. Methods A378 (1996) 221.

[12] A. Artamonov and P. Gorbunov, CHORUS Internal Note 97029, 23 February 1998, updated on 12 March 1999.

[13] M.G. van Beuzekom et al., Nucl. Instr. and Meth. A427 (1999) 587.

[14] J. Brunner with contributions from M. van der Donckt, D. Cussans, H. Meinhard, CHORAL Manual, revision 1.3, 1998; program version 2.19/03.

[15] I. Tsukerman, CHORUS Internal Note 98011, 14 September 1998.

[16] R.G.C. Oldeman, CHORUS Internal Note 97020, 21 September 1997.

[17] E. Eichten, I. Hinchliffe, K. Lane and C. Quigg, Rev. Mod. Phys. 56 (1984) 579; Erratum: 58 (1986) 1065.

[18] C. Peterson et al., Phys. Rev. D27 (1983) 101.

[19] C.A.F.J. van der Poel, PhD thesis, University of Nijmegen, Nijmegen, The Netherlands, 1999.

[20] C. Caso et al., Particle Data Group, Eur. Phys. J. C3 (1998) 1.

[21] CERN Program Library Long Writeup Y250, HBOOK Reference Manual, version 4.22, subroutine HMCMLL.

[22] R.G.C. Oldeman, PhD thesis, University of Amsterdam, Amsterdam, The Netherlands, 2000.

[23] R.G.C. Oldeman, CHORUS Internal Note 98001, 14 March 1998.

[24] I. Tsukerman, PhD thesis, ITEP, Moscow, Russia, 2000 (in preparation).

[25] T. Weiler, Phys. Rev. Lett. 44 (1980) 304.

[26] M. de Jong, PhD thesis, University of Amsterdam, Amsterdam, The Netherlands, 1991.

[27] J.G.H. de Groot et al., CDHS Collaboration, Z. Phys. C1 (1979) 143. 